\title{
The distance indicators in gamma-ray pulsars
}

\author{
Wei Wang \\ National Astronomical Observatories, Chinese Academy of Sciences, Beijing 100012, China \\ email: wangwei@bao.ac.cn
}

\begin{abstract}
Distance measurements of gamma-ray pulsars are challenging questions in present pulsar studies. The Large Area Telescope (LAT) aboard the Fermi gamma-ray observatory discovered more than 100 gamma-ray pulsars, including 34 new gamma-selected pulsars which nearly have no distance information. We study the relation between gamma-ray emission efficiency $\left(\eta=L_{\gamma} / \dot{E}\right)$ and pulsar parameters, for young radio-selected gamma-ray pulsars with known distance information. We have introduced three generation order parameters to describe gamma-ray emission properties of pulsars, and find a strong correlation between $\eta$ and $\zeta_{3}$, the generation order parameter which reflects $\gamma$-ray photon generations in pair cascade processes induced by magnetic field absorption in pulsar magnetosphere. A good correlation between $\eta$ and $B_{\mathrm{LC}}$, the magnetic field at the light cylinder radius, is also found. These correlations can serve as distance indicators in gamma-ray pulsars, to evaluate distances for gamma-selected pulsars. Distances of 35 gamma-selected pulsars are estimated, which could be tested by other distance measurement methods. The physical origin of the correlations may be also interesting for pulsar studies.
\end{abstract}

Keywords. gamma rays: general - pulsars: general - stars: neutron

\section{Introduction}

The distance measurement of pulsars is always a difficult problem in pulsar studies. Trigonometric parallax measurements of radio pulsars are the reliable method, but are only available for the nearby pulsars $(<0.4 \mathrm{kpc})$. The most common way to obtain radio pulsar distance is based on the computation from dispersion measurement (DM) coupled to an electron density distribution model like NE 2001 model (Cordes \& Lazio 2002). The pulsar distance can be also estimated from kinematic model: the distance of possible associated objects (supernova remnants, star clusters, or HII regions) could be measured from Doppler shift of absorption or emission lines in HI spectrum together with the rotation curve model of the Galaxy. The distance of some pulsars with X-ray emissions can be estimated from X-ray observations of the absorbing column or from correlations in X-ray luminosities versus spin-down power or photon index (Gotthelf 2003; Wang 2009). These methods may be available for radio or X-ray pulsars, but for gamma-selected pulsars if no possible associated objects, we would have no any information on their distance.

Presently more than 100 gamma-ray pulsars are discovered by the Fermi/LAT, including 35 gamma-selected pulsars. Distance information for these gamma-ray pulsars is important for pulsar physics studies. Based on the EGRET pulsars, Thompson et al. (1999) found a possible correlation of $L_{\gamma} \propto \dot{E}^{1 / 2}$. For the larger sample of gamma-ray pulsars (Abdo et al. 2010), the young pulsars looks to still follow this relation with a large scattering factors of more than 10. Thus a better correlation in gamma-ray pulsars should be probed for distance estimate for gamma-ray pulsars. 
Table 1. The estimated distances of 35 gamma-selected pulsars. $d_{1-2}$ denotes the distance calculated from relations of $\eta-\zeta_{3}$ and $\eta-B_{\mathrm{LC}}$, respectively. $d_{3}$ is the estimated distance from other methods with references provided.

\begin{tabular}{|c|c|c|c|c|c|c|c|}
\hline Pulsar & $\begin{array}{l}P \\
\mathrm{~s}\end{array}$ & $\begin{array}{c}\dot{P} \\
\mathrm{~s} \mathrm{~s}^{-1}\end{array}$ & $\begin{array}{c}F_{\gamma}(>100 \mathrm{MeV}) \\
\quad \text { erg } \mathrm{cm}^{-2} \mathrm{~s}^{-1}\end{array}$ & $\begin{array}{c}d_{1} \\
\mathrm{kpc}\end{array}$ & $\begin{array}{c}d_{2} \\
\mathrm{kpc}\end{array}$ & $\begin{array}{l}d_{r f} \\
\mathrm{kpc}\end{array}$ & reference \\
\hline $\mathrm{J} 0007+7303$ & 0.316 & $3.61 \times 10^{-13}$ & $3.82 \times 10^{-10}$ & $0.86_{-0.32}^{+0.30}$ & $1.18^{+0.72}$ & $1.4 \pm 0.3$ & Pineault et al. 1993 \\
\hline $\mathrm{J} 0106+4855$ & 0.083 & $4.3 \times 10^{-16}$ & $2.4 \times 10^{-11}$ & $0.92_{-0.35}^{+0.33}$ & $1.25_{-0.52}^{+0.44}$ & & \\
\hline $\mathrm{J} 0357+32$ & 0.444 & $1.20 \times 10^{-14}$ & $6.38 \times 10^{-11}$ & $0.72_{-0.29}^{+0.25}$ & $0.73_{-0.30}^{+0.51}$ & & \\
\hline $\mathrm{J} 0622+3749$ & 0.333 & $2.5 \times 10^{-14}$ & $1.69 \times 10^{-11}$ & $2.33_{-0.65}^{+0.73}$ & $2.73_{-0.89}^{+1.21}$ & & \\
\hline $\mathrm{J} 0633+0632$ & 0.297 & $7.95 \times 10^{-14}$ & $8.00 \times 10^{-11}$ & $1.26_{-0.48}^{+0.41}$ & $1.37_{-0.60}^{+0.76}$ & & \\
\hline $\mathrm{J} 0633+1746$ & 0.237 & $1.10 \times 10^{-14}$ & $3.38 \times 10^{-9}$ & $0.19_{-0.07}^{+0.08}$ & $0.17_{-0.06}^{+0.09}$ & $0.25_{-0.06}^{+0.12}$ & Faherty et al. 2007 \\
\hline J1418-5819 & 0.111 & $1.70 \times 10^{-13}$ & $2.35 \times 10^{-10}$ & $1.39_{-0.57}^{+0.58}$ & $1.86_{-0.80}^{+1.09}$ & $2-5$ & $\mathrm{Ng}$ et al. 2005 \\
\hline $\mathrm{J} 1459-60$ & 0.103 & $2.55 \times 10^{-14}$ & $1.06 \times 10^{-10}$ & $1.76_{-0.67}^{+0.70}$ & $1.62_{-0.69}^{+0.80}$ & & \\
\hline $\mathrm{J} 1620 \mathrm{C} 4927$ & 0.171 & $1.0 \times 10^{-14}$ & $1.35 \times 10^{-10}$ & $\begin{array}{l}0.79_{-0.34}^{0.35} \\
0.07\end{array}$ & $0.97_{-0.35}^{+0.59}$ & & \\
\hline J1732-31 & 0.197 & $2.62 \times 10^{-14}$ & $2.42 \times 10^{-10}$ & $0.77_{-0.35}^{+0.41}$ & $0.86_{-0.30}^{+0.49}$ & & \\
\hline $\mathrm{J} 1741-2054$ & 0.414 & $1.69 \times 10^{-14}$ & $1.28 \times 10^{-10}$ & $0.59_{-0.25}^{+0.26}$ & $0.80_{-0.29}^{+0.48}$ & $0.38 \pm 0.11$ & Camilo et al. 2009 \\
\hline J1746C 3239 & 0.2 & $6.6 \times 10^{-15}$ & $7.86 \times 10^{-11}$ & $0.75_{-0.30}^{+0.27}$ & $1.06_{-0.42}^{+0.62}$ & & \\
\hline $\mathrm{J} 1803 \mathrm{C} 2149$ & 0.106 & $1.9 \times 10^{-14}$ & $1.31 \times 10^{-10}$ & $1.01_{-0.49}^{+0.43}$ & $1.42_{-0.60}^{+0.91}$ & & \\
\hline J1809-2332 & 0.147 & $3.44 \times 10^{-14}$ & $4.13 \times 10^{-10}$ & $0.78_{-0.31}^{+0.31}$ & $0.81_{-0.30}^{+0.00}$ & $1.7 \pm 1.0$ & Oka et al. 1999 \\
\hline J1813-1246 & 0.048 & $1.76 \times 10^{-14}$ & $1.69 \times 10^{-10}$ & $2.18_{-0.64}^{+0.71}$ & $1.56_{-0.68}^{+1.21}$ & & \\
\hline J1826-1256 & 0.110 & $1.21 \times 10^{-13}$ & $3.34 \times 10^{-10}$ & $1.29_{-0.44}^{+0.56}$ & $1.39_{-0.60}^{+0.08}$ & & \\
\hline $\mathrm{J} 1836+5925$ & 0.173 & $1.49 \times 10^{-15}$ & $5.99 \times 10^{-10}$ & $0.32_{-0.14}^{+0.44}$ & $0.27_{-0.09}^{+0.60}$ & $<0.8$ & Halpern et al. 2007 \\
\hline J1838-0537 & 0.146 & $4.6 \times 10^{-13}$ & $1.75 \times 10^{-10}$ & $2.18_{-0.69}^{+0.74}$ & $2.62_{-0.90}^{+1.39}$ & & \\
\hline $\mathrm{J} 1907+0602$ & 0.107 & $8.68 \times 10^{-14}$ & $2.75 \times 10^{-10}$ & $\begin{array}{r}-0.69 \\
1.39_{-0.40}^{+0.46}\end{array}$ & $1.42_{-0.61}^{+0.95}$ & & \\
\hline $\mathrm{J} 1958+2846$ & 0.290 & $2.10 \times 10^{-13}$ & $8.45 \times 10^{-11}$ & $1.54_{-0.51}^{+0.56}$ & $1.86_{-0.78}^{+1.01}$ & & \\
\hline $\mathrm{J} 2021+4026$ & 0.265 & $5.48 \times 10^{-14}$ & $9.76 \times 10^{-10}$ & $0.38_{-0.21}^{+0.20}$ & $0.46_{-0.18}^{+0.20}$ & $1.5 \pm 0.5$ & Landecker et al. 1980 \\
\hline $\mathrm{J} 2028+3332$ & 0.177 & $4.9 \times 10^{-15}$ & $6.09 \times 10^{-11}$ & $0.80_{-0.31}^{+0.32}$ & $1.17_{-0.52}^{+0.59}$ & & \\
\hline $\mathrm{J} 2030+4415$ & 0.227 & $6.5 \times 10^{-15}$ & $7.06 \times 10^{-11}$ & $0.76_{-0.30}^{+0.31}$ & $1.03_{-0.50}^{+0.52}$ & & \\
\hline $\mathrm{J} 2032+4127$ & 0.143 & $1.98 \times 10^{-14}$ & $1.11 \times 10^{-10}$ & $1.32_{-0.52}^{+0.49}$ & $1.33_{-0.50}^{+0.71}$ & $1.6-3.6$ & Camilo et al. 2009 \\
\hline $\mathrm{J} 2238+59$ & 0.163 & $9.86 \times 10^{-14}$ & $5.44 \times 10^{-11}$ & $2.36_{-0.70}^{+0.75}$ & $2.64_{-0.93}^{+1.36}$ & & \\
\hline $\mathrm{J} 1023-5746$ & 0.111 & $3.84 \times 10^{-13}$ & $2.69 \times 10^{-10}$ & $1.77_{-0.55}^{+0.70}$ & $\begin{array}{r}-0.93 \\
2.09_{-0.88}^{+0.95}\end{array}$ & & \\
\hline J1044-5737 & 0.139 & $5.46 \times 10^{-14}$ & $1.03 \times 10^{-10}$ & $\begin{array}{r}-0.55 \\
1.72_{-0.65}^{+0.60}\end{array}$ & $\begin{array}{r}-0.88 \\
1.86_{-0.72}^{+0.86}\end{array}$ & & \\
\hline J1413-6205 & 0.110 & $2.78 \times 10^{-14}$ & $1.29 \times 10^{-10}$ & $2.52_{-0.92}^{+0.89}$ & $1.56_{-0.60}^{+1.12}$ & & \\
\hline J1429-5911 & 0.116 & $3.05 \times 10^{-14}$ & $9.26 \times 10^{-11}$ & $1.84_{-0.69}^{+0.64}$ & $1.79_{-0.70}^{+0.07}$ & & \\
\hline $\mathrm{J} 1846+0919$ & 0.226 & $9.92 \times 10^{-15}$ & $3.58 \times 10^{-11}$ & $1.52_{-0.70}^{+0.69}$ & $1.44_{-0.51}^{+0.80}$ & & \\
\hline $\mathrm{J} 1954+2836$ & 0.093 & $2.12 \times 10^{-14}$ & $9.75 \times 10^{-11}$ & $\begin{array}{r}-0.70 \\
1.90_{-0.80}^{+0.67}\end{array}$ & $\begin{array}{r}-0.51 \\
1.72_{-0.71}^{+1.10}\end{array}$ & & \\
\hline $\mathrm{J} 1957+5033$ & 0.375 & $7.08 \times 10^{-15}$ & $2.27 \times 10^{-11}$ & $1.22_{-0.45}^{+0.41}$ & $1.31_{-0.37}^{+0.65}$ & & \\
\hline $\mathrm{J} 2055+2500$ & 0.320 & $4.08 \times 10^{-15}$ & $1.15 \times 10^{-10}$ & $0.56_{-0.23}^{+0.19}$ & $0.61_{-0.19}^{+0.30}$ & & \\
\hline $\mathrm{J} 2111+4606$ & 0.158 & $1.4 \times 10^{-13}$ & $4.13 \times 10^{-11}$ & $2.79_{-1.01}^{+0.90}$ & $3.70_{-1.70}^{+1.59}$ & & \\
\hline $\mathrm{J} 2139+4716$ & 0.282 & $1.8 \times 10^{-15}$ & $2.51 \times 10^{-11}$ & $0.81_{-0.31}^{+0.32}$ & $1.08_{-0.50}^{+0.54}$ & & \\
\hline
\end{tabular}

\section{Distance Indicators in Gamma-ray Pulsars}

Gamma-ray emission efficiency $\left(\eta=L_{\gamma} / \dot{E}\right)$ is an important parameter in gamma-ray pulsars, which varies for different populations of pulsars. With the gamma-ray pulsar sample with distance information, we have studied the possible relations between the efficiency and some pulsar parameters, like spin period, age, magnetic field at light cylin$\operatorname{der}\left(B_{\mathrm{LC}}\right)$, and three generation order parameters $\left(\zeta_{1-3}\right.$, see details in Wang \& Zhao 2004). We found that there exist strong correlations between $\eta$ and $B_{\mathrm{LC}}$ and $\zeta_{3}$ (Wang 2011). With these correlations, we have a possible way to estimate a reliable distance for gamma-ray pulsars with only known $P, \dot{P}$ and $F_{\gamma}$.

We use the distance indicators obtained by $\eta-\zeta_{3} B_{\mathrm{LC}}$ correlations to estimate the distances of the 35 gamma-selected pulsars which nearly have no other distance information (Table 1 ). The evaluated distances by $\eta-\zeta_{3}$ and $\eta-B_{\mathrm{LC}}$ correlations are similar, suggesting that two distance indicators can be checked by each other. 


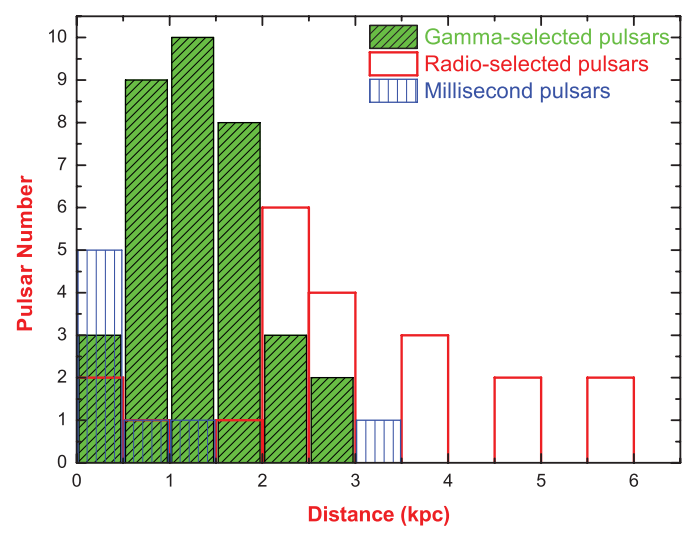

Figure 1. The distance distributions of three classes of gamma-ray pulsars.

The distance of some gamma-selected pulsars was also estimated by other methods, which can compared with our estimate. For the Geminga pulsar, we estimate the distance of $0.19 \pm 0.07 \mathrm{kpc}$ which is well consistent with the distance value of $0.25_{-0.06}^{+0.12} \mathrm{kpc}$ from the optical trigonometric parallax measurement (Faherty et al. 2007). For PSR J1836+5925, we estimate its distance as $\sim 0.3 \mathrm{kpc}$ (corresponding to an efficiency of $\sim 55 \%$ ) which is also well below the upper limits of $0.8 \mathrm{kpc}$ according to its thermal X-ray spectrum (Halpern et al. 2007). For other gamma-selected pulsars, our estimated distance values are generally below those from other methods, but may be more reliable with the gamma-ray efficiency $\eta$ generally below 1 .

Fig 1. shows the distance distributions of three classes of gamma-ray pulsars: gammaselected pulsars, radio-selected pulsars and millisecond pulsars. The distances of gammaselected pulsars are provided by the distance indicator of the $\eta-\zeta_{3}$ relation. Gamma-ray loud millisecond pulsars distribute at a distance peak around $0.3 \mathrm{kpc}$ because MSPs generally have lower spin-down powers. Gamma-selected young pulsars distribute at the distance peak of $\sim 1.2 \mathrm{kpc}$, while radio-selected young pulsars distribute at the distance peak of $\sim 2.5 \mathrm{kpc}$. This difference in distance distributions for two classes of gamma-ray young pulsars may involve further interest. The nearby unresolved radio-quiet gammaray pulsars could contribute to diffuse gamma-ray/positron background specially for the high-latitude pulsars located in the Gould Belt (Wang et al. 2005).

\section{References}

Abdo, A. A. et al. 2010, ApJS, 187, 460

Camilo, F., et al. 2009, ApJ, 705, 1

Cordes, J. M. \& Lazio, T. J. W. 2002, astro-ph/0207156

Faherty, J., Walter, F. M., \& Anderson, J. 2007, ApESSS, 308, 225

Gotthelf, E. V. 2003, ApJ, 591, 361

Landecker, T. L., Roger, R. S., \& Higgs, L. A. 1980, A\&AS, 39, 133

Ng, C.-Y., Roberts, M. S. E., \& Romani, R. W. 2005, ApJ, 627, 904

Oka, T. et al. 1999, ApJ, 526, 764

Pineault, S. et al. 1993, AJ, 105, 1060

Thompson, D. J., et al. 1999, ApJ, 516, 297

Wang, W. \& Zhang, Y. 2004, ApJ, 601, 1038

Wang, W., Jiang, Z. J., Pun, C. S. J., \& Cheng, K. S. 2005, MNRAS, 360, 646

Wang, W. 2009, RAA, 9, 1241

Wang, W. 2011, RAA, 11, 824 